\title{
Vínculo Afectivo Madres - Hijos Neonatos a Término según Edad y paridad: Una Comparación
}

\section{A comparison of the affective link between mothers and their term-born children, depending on parity and}

Gladys Vanessa Mite Cárdenas ${ }^{1, *}$, Myriam Patricia Pardo Torres ${ }^{1}$

INFORMACIÓN DEL

ARTÍCULO

Fecha de recepción: 1 de mayo de 2018.

Fecha de aceptación: 4 de septiembre de 2018 .
1 Universidad Nacional de Colombia. Sede Bogotá, Facultad de Enfermería.

*Autor de correspondencia: Gladys Vanessa Mite Cárdenas, Universidad Nacional de Colombia, Facultad de Enfermería, Ciudad Universitaria, Cra 30, calle 45. Edificio 228. Bogotá, Colombia.

Correo electrónico: gvmitec@unal.edu.co Telf.0991854667

ENLACE DOI:

http://dx.doi.org/10.31095/investigatio. 2019.12.4

\section{Resumen}

Este trabajo está enmarcado en el área temática de cuidado materno perinatal. El objetivo es comparar el vínculo afectivo de madres con sus hijos recién nacidos a término según edad y paridad. Se utiliza una investigación cuantitativa y descriptiva en dos fases. La primera fase es la validez facial del instrumento, la segunda fase de la investigación con una muestra de 157 díadas madre-hijo, con el grupo de adolescentes y adultas primíparas y multíparas. Los resultados se analizaron con el paquete estadístico SPSS, además se complementó con el estado del arte. Los resultados arrojan diferencia significativa entre los tres grupos de comparación con una mayor puntuación para el vínculo afectivo en madres primíparas seguidos de madres multípararas en relación con madres adolescentes quienes además presentaron un valor menor de (Kruskal-Wallis) $=0,04$ y 0,02 respectivamente en la dimensión de apoyo emocional y unión interacción dimensiones del Instrumento utilizado.

\section{Palabras Clave:}

Lactancia materna; madre adolescente; madre adulta; neonato a término; rol maternal; vínculo afectivo.

Clasificación JEL: J13.

\section{Abstract}

This work's framework is the thematic area of perinatal maternal care. It purports to compare the affective bond that exists between mothers and their term-born babies, depending on parity and mother's age. A two-phase quantitative and descriptive research was made; the first phase ascertained the instrument's face validity. A total of 168 mothers participated in this phase; comprehensibility was above $90 \%$; the second research phase sampled 157 mother-child dyads, with new term-born healthy children. Mothers' age was between 15 and 19 for the adolescent group; it was 20 or over for adult women who had given birth either for the first time, or otherwise. The SPSS statistical package was used to analyze results and compare the groups and the instrument's dimensions. This was complemented with the available state of the art. Results show significant differences in the three comparison groups with a higher affective scores for first-time mothers followed by several-times mothers in relation to

$$
67
$$

NVESTIGATIO No. 12, marzo 2019, pp. 67-78,

ISSN: 1390 - 6399・ISSN-e: 2602 - 8336 


\section{Introducción}

La capacidad del ser humano de establecer relaciones efectivas y duraderas con sus semejantes lleva a realizar actividades que se convierten en una necesidad para vivir en la sociedad contemporánea; el vínculo afectivo entre la madre y su hijo se inicia desde que el feto se encuentra en el vientre materno y continúa al nacimiento. Este vínculo es expresado con gestos de cariño, y que puede ser diferente por la idiosincrasia de cada mujer de acuerdo a sus características biologías o sociales que influyan. El vínculo afectivo entre madre e hijo es esencial para el niño, que brinda seguridad y confianza, necesarios para el desarrollo y aprendizaje. John Bowlby quien es el autor de la Teoría del Apego (1907-1990) lo define como "un conjunto de conductas del niño en la búsqueda de proximidad con una o varias personas adultas, denominadas «figuras de apego»" (Fernández, 2013, p.5). Este vínculo es vital no solo para el desarrollo del aprendizaje sino de la formación de la personalidad específicamente del temperamento, de allí si los niños tienen más $o$ menos herramientas para confrontar el medio que les rodea. El vínculo afectivo también se define como "la capacidad humana de establecer lazos afectivos con otros seres humanos [...] Estos afectos se ven estimulados con la presencia o ausencia del otro, con la disponibilidad o indisponibilidad del otro" (Fernández, 2013, p.2).

De igual modo, desde el área disciplinar de la enfermería, en el modelo de Adopción del rol materno de Mercer: convertirse en madre, se refiere a la unión como un proceso en el que se crea un vínculo emocional que establece la madre con su hijo a través de conductas que hacen parte de las características de la madre al interactuar con el mismo, y que a su vez constituyen la identidad maternal (Meighan, 2011, p. 581-598)

Se describen en la literatura algunos factores que interfieren en el establecimiento de la vinculación afectiva. Los más importantes son: la edad de la madre, la presencia de redes de apoyo, procedencia de la madre, la vía del parto, el contacto temprano piel a piel, lactancia materna, ajustes psicológicos cognitivos de la formación y desarrollo de la personalidad, aspectos sociales y la adolescencia, en especial la tardía. Estos factores demuestran diferencias en el reconocimiento de la nueva identidad del rol parental (Garrido y Marchán, 2011).

Se ha demostrado en varios estudios cómo el vínculo se manifiesta en las madres adultas de manera más segura y estable frente a las madres adolescentes. El estudio de Crugnola et al. (2014) concluye que el apego materno inseguro en las díadas con madres adolescentes (frente a la diada madre adulta infante) es más influyente como factor de riesgo sobre la vinculación afectiva. Relacionando los aspectos biológicos y psicológicos en desarrollo de la adolescente al enfrentar el rol maternal siendo un ciclo normal en la madre adulta en la sociedad. Carrillo y Quispe (2013) afirman que las mujeres adultas tienen un acercamiento seguro y estable emocionalmente con sus hijos en relación 
con las madres adolescentes. Por otra parte, Rosero y Sandoval (2016) establecen que las madres adultas multíparas manifestaron una interacción armónica y una tonalidad afectiva placentera, aun siendo el primer contacto con su bebé después de 25 horas del parto. Del mismo modo un estudio realizado con mujeres adultas en el primer embarazo demuestra que $88,3 \%$ presentaron un puntaje de ajuste emocional adecuado Farkas et al. (2008); lo que se puede evidenciar que en la adultez predomina una estabilidad emocional desde el embarazo y continua hasta el nacimiento del bebé. Al respeto con lo mencionado se afirma que entre más seguro es el apego menos sintomatología ansiosa-depresiva, menos problemas en las relaciones interpersonales y en el rol social, y un mayor ajuste socioemocional. Esto se manifiesta con un pensamiento con más claridad y un apego seguro y una independencia de las madres adultas.

Sánchez Martínez y Hernández, (2004) realizaron un estudio con madres adultas multíparas, y encontraron que las madres valoraban los comportamientos que muestran vinculación con el recién nacido; por ejemplo, el disfrute del contacto con su bebé, lo cual califican como "beneficioso". Los resultados revelan una relación significativa entre el grado de satisfacción materna y el tiempo de contacto piel a piel con su hijo al nacer. Los estudios dirigidos al vínculo afectivo son de importancia para la comunidad académica y para el ejercicio práctico, debido a los efectos negativos y a los trastornos hacia el futuro; además que un vínculo precoz posparto es satisfactorio para las madres y beneficioso para los bebes (Sánchez et al., 2004). Por estas razones, y enfocándose en el bienestar social y emocional del niño, es necesario fortalecer la relación vincular madre-hijo.

La Organización Panamericana de la Salud y la Organización Mundial de la Salud recomiendan, realizar programas que promocionen la salud materna y el desarrollo emocional e intelectual del niño (OPS - OMS, 2016). Siendo el posparto un momento de interés para los profesionales de salud, al ofrecer a la diada madre e hijo intervenciones de cuidado dirigidas al fortalecimiento del vínculo afectivo a partir de las necesidades identificadas (Moreno et al., 2014).

En Ecuador se acogen políticas y estrategias generales, como el Plan Nacional para el buen vivir 2013-2017 y los Objetivos de Desarrollo Sostenible (ODS), que abordan la salud sexual y reproductiva, la lactancia materna y el embarazo adolescente. Así mismo, se han establecido normas que van dirigidas a fortalecer el apego precoz madre-neonato y propiciar los beneficios emocionales con menos estrés y mayores sentimientos de confianza y competencia para el recién nacido, lo cual surge a partir del contacto piel a piel y del inicio de la lactancia materna justo después del nacimiento.Es asi que el presente protocolo tiene como objetivo asegurar el vínculo madre-hijo o hija desde el nacimiento (Ministerio de Salud Pública del Ecuador, 2010).

\section{Materiales y métodos}

Estudio cuantitativo, descriptivo, 
realizado en dos fases. La primera fase: validez facial del instrumento Vínculo entre padres e hijos neonatos en el contexto ecuatoriano, con la participación de 168 personas con bajo nivel de escolaridad, acogiéndose al principio de parsimonia, el resultado de este proceso arrojó una comprensibilidad por encima de $90 \%$ para el instrumento global y para cada uno de los ítems (Vargas, 2016).

La segunda fase de la investigación indagó el fenómeno Vínculo afectivo entre padres e hijos neonatos a un total de 157 díadas de puérperas con sus hijos recién nacidos a término sanos, distribuidas en tres grupos uno de madres adolescentes primíparas con edades entre 15 y 19 años, y los otros dos correspondían a madres adultas primíparas y multíparas de 20 años y más, usuarias pertenecientes al Ministerio de Salud Pública y al Instituto de Seguridad Social (IESS) de la Provincia BolívarEcuador.

Los criterios de inclusión fueron: madres en puerperio por parto vaginal, alfabetizadas captadas en las 48 horas siguientes al parto. Como criterios de exclusión se tuvieron: madres e hijos que en el transcurso de la investigación presentaran complicaciones, puérperas con trastorno mental o de aprendizaje, parto por cesárea.

La variable Vínculo entre padres e hijos neonatos de Vargas (2016) fue medida con el instrumento que contiene 24 ítems y una escala de medición tipo Likert con cinco posibilidades de respuesta. El análisis factorial mediante la rotación Varimax agrupó los ítems en cuatro factores a saber: Apoyo emocional (ítems 6); Unión - interacción (ítems: 7); Apoyo informativo (ítems: 6) y Estrés (items: 5). El instrumento reporta una validez de constructo mediante el Test de Káiser-Meyer-Olkin (KMO) de 0,732; y una consistencia interna de 0,69.

\section{Procedimiento metodológico}

Bajo los preceptos éticos de las normas de la Council for International Organizations of Medical Sciences -CIOMS- (2016) de investigación con seres humanos y la Resolución 8430 (República de Colombia, Ministerio de Salud, 1993, p. 8), y con el Aval 0059 del 2016 del Comité de Ética en Investigación Científica de la Facultad de Enfermería de la Universidad Nacional de Colombia, se desarrolló el protocolo entre el 17 de diciembre de 2016 y el 9 de abril de 2017 con un solo momento de medición en cada una de las dos fases del estudio. Se propone las siguientes hipótesis estadísticas para la investigación:

-Hipótesis alterna 1: Existe diferencia en el vínculo afectivo de las madres adolescentes con sus hijos recién nacidos a término comparado con el de las madres adultas con sus hijos recién nacidos a término.

-Hipótesis nula 1: No existe diferencia en el vínculo afectivo de las madres adolescentes con sus hijos recién nacidos a término comparado con el de las madres adultas con sus hijos recién nacidos a término.

-Hipótesis alterna 1: Existe diferencia en el vínculo afectivo de las madres 
primíparas con sus hijos recién nacidos a término comparado con el de las madres multíparas con sus hijos recién nacidos a término.

-Hipótesis nula 1: No existe diferencia en el vínculo afectivo de las madres primíparas con sus hijos recién nacidos a término comparado con el de las madres multíparas con sus hijos recién nacidos a término.

\section{Resultados}

Con respecto a las características sociodemográficas de las participantes, el grupo de adolescentes de 15 y 19 años se relaciona con las estadísticas globales del embarazo adolescente en el Ecuador, con una mayor proporción en el grupo 17-19 años (UTPL: ILFAM; ORIENTAR, 2016).

Vinculo afectivo madre-neonato en general y por dimensiones del instrumento Vinculo entre padres e hijos neonatos

La interpretación del resultado total es: grado bajo de vinculación afectiva 85 puntos o menos; grado medio entre $86 \mathrm{y}$ 89 puntos y grado alto 90 o más puntos. En la comparación de las medias del vínculo afectivo madres e hijos de los tres grupos de las diadas de adolescentes, adultas primíparas y adultas multíparas se puede afirmar que existe una diferencia significativa en la prueba de Krusskal Wallis para muestras independientes dada por un $P$ de 0,022 , con desventaja para el grupo de adolescentes.

La Tabla 1 muestra los estadígrafos de los tres grupos. Al compararlos, vislumbra que la media más alta del vínculo afectivo se encuentra en el grupo de adultas primíparas $(116,4)$, seguido de las adultas multíparas $(115,4)$ y de las adolescentes $(113,3)$, con una mayor varianza en este último grupo. Existe una asimetría en la distribución con tendencia negativa en los tres grupos.

Así pues, la curtosis es útil porque demuestra que la concentración de los valores del vínculo afectivo alrededor de la zona central de la distribución de frecuencias está a favor de las adultas

Tabla 1 .

Estadígrafos del análisis del valor total del vínculo afectivo del estudio Vínculo afectivo madres - hijos neonatos a término según edad y paridad: una comparación.

\begin{tabular}{|c|c|c|c|c|c|c|c|}
\hline Grupos & Media & Varianza & $\begin{array}{l}\text { Desviación } \\
\text { típica }\end{array}$ & $\begin{array}{l}\text { Valor } \\
\text { mínimo }\end{array}$ & $\begin{array}{l}\text { Valor } \\
\text { máximo }\end{array}$ & Curtosis & Asimetría \\
\hline $\begin{array}{l}\text { Adolecentes } \\
\qquad(\mathrm{n}=53)\end{array}$ & 113,3 & 41,3 & 6,4 & 90 & 120 & 2,0 & $-1,2$ \\
\hline $\begin{array}{l}\text { Adultas } \\
\text { Primíparas } \\
(\mathrm{n}=53)\end{array}$ & 116,4 & 21,5 & 4,6 & 99 & 120 & 3,9 & $-1,9$ \\
\hline $\begin{array}{l}\text { Adultas } \\
\text { multíparas } \\
(\mathrm{n}=53)\end{array}$ & 115,4 & 23,5 & 4,8 & 94 & 120 & 7,0 & $-2,2$ \\
\hline
\end{tabular}

\section{1}

INVESTIGATIO No. 12, marzo 2019, pp. 67-78, ISSN: 1390 - 6399• ISSN-e: 2602 - 8336 


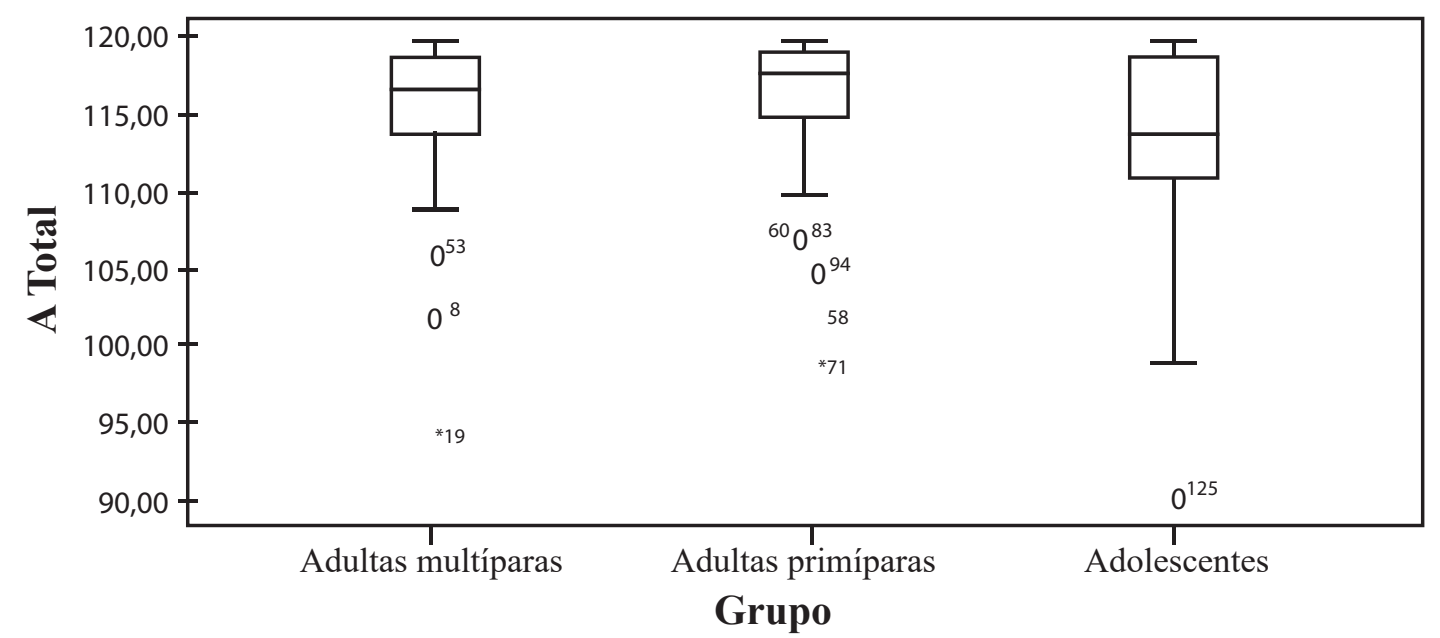

Figura 1. A total= Comparación del vínculo afectivo madres-hijos en los grupos adolescentes, adultas primíparas y adultas multíparas, Provincia de Bolívar.

multíparas, cuyo valor es mayor, seguido de las adultas primíparas. Esto es posible visualizarlo gráficamente en la Figura 1.

Con relación a la comparación de las medias de las dimensiones del instrumento Vínculo Afectivo madres-hijo, la prueba de hipótesis muestra una significancia estadística en las dimensiones Apoyo emocional y Unión interacción, descrito a continuación:

En la dimensión Apoyo emocional de los grupos seleccionados adolescentes, adultas primíparas y adultas multíparas, la prueba de Krusskal-Wallis para muestras independientes rechaza la hipótesis nula, por tanto, se puede afirmar que existe una diferencia significativa con un $P$ de 0,04 y una visible desventaja en el grupo de adolescentes.

La Tabla 2 presenta los resultados de los estadígrafos de los tres grupos que, al compararlos, permite concluir que la media más alta de la dimensión Apoyo

Tabla 2.

Estadígrafos del análisis de la dimensión Apoyo emocional del vínculo afectivo madres-hijos, Ecuador, Provincia de Bolívar.

\begin{tabular}{|c|c|c|c|c|c|c|c|}
\hline Grupos & Media & Varianza & $\begin{array}{l}\text { Desviación } \\
\text { típica }\end{array}$ & $\begin{array}{l}\text { Valor } \\
\text { mínimo }\end{array}$ & $\begin{array}{l}\text { Valor } \\
\text { máximo }\end{array}$ & Curtosis & Asimetría \\
\hline $\begin{array}{l}\text { Adolecentes } \\
\qquad(\mathrm{n}=53)\end{array}$ & 93,2 & 62,2 & 7,9 & 58,3 & 100 & 5,9 & $-1,9$ \\
\hline $\begin{array}{l}\text { Adultas } \\
\text { Primíparas } \\
(\mathrm{n}=53)\end{array}$ & 95,9 & 40,9 & 6,4 & 70,8 & 100 & 5,5 & $-1,6$ \\
\hline $\begin{array}{l}\text { Adultas } \\
\text { multíparas } \\
(\mathrm{n}=53)\end{array}$ & 95,8 & 31,4 & 5,6 & 75 & 100 & 2,6 & $-2,2$ \\
\hline
\end{tabular}




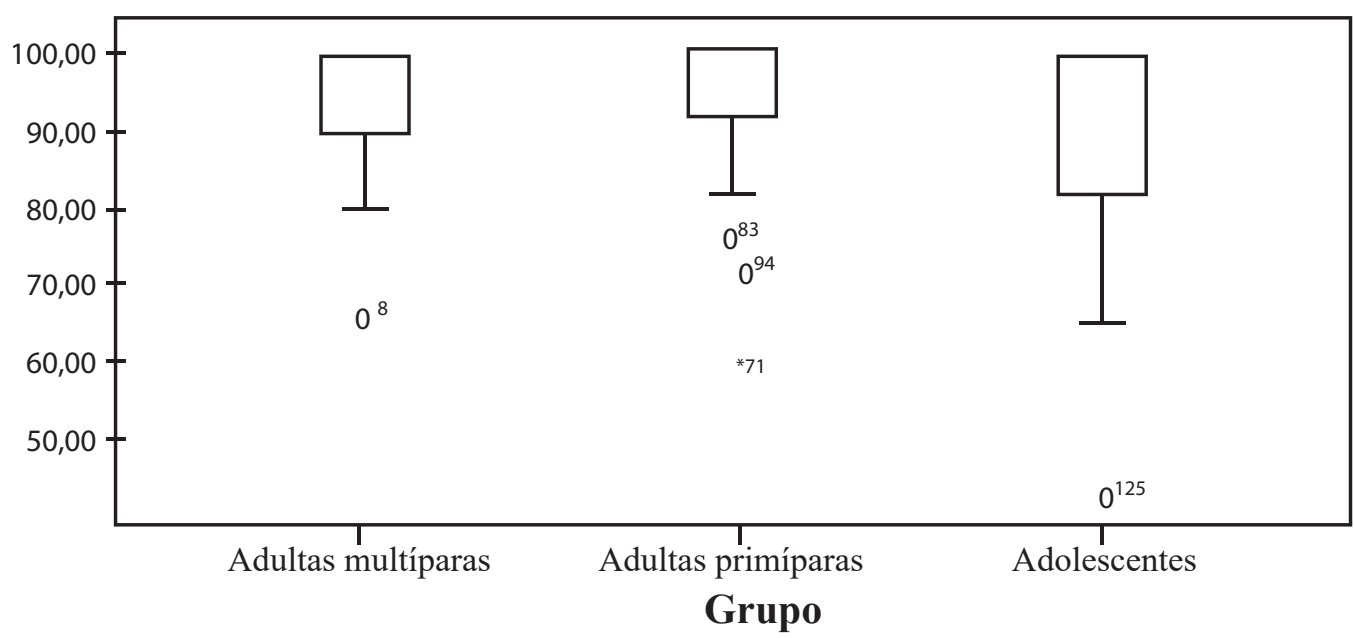

Figura 2. Comparación de la dimensión Apoyo Emocional del vínculo afectivo madres-hijos en los grupos adolescentes, adultas primíparas y adultas multíparas, Provincia de Bolívar.

emocional del vínculo afectivo madre-hijo se encuentra en el grupo de adultas primíparas, seguido por adultas multíparas y adolescentes, con una mayor varianza en este último grupo.

En el análisis estadístico de la dimensión Apoyo emocional en la Figura 2, aparece la posición de la media en los tres grupos con una desventaja en el grupo de adolescentes y una mayor dispersión de los datos en comparación con el de las adultas.
Por otra parte, en la dimensión Unión-interacción, el resultado de la prueba de Krusskal-Wallis reporta un $P$ de 0.020 , a partir de este resultado, es posible afirmar que en la dimensión. Unión-interacción existe una diferencia significativa en la comparación de las medias en los grupos seleccionados adolescentes, adultas primíparas y adultas multíparas.

Como se observa en la Tabla 3, los resultados en la dimensión Unión-interacción

Tabla 3.

Estadígrafos del análisis de la dimensión Unión-interacción del vínculo afectivo madres-hijos, Ecuador, Provincia de Bolívar.

\begin{tabular}{|c|c|c|c|c|c|c|c|}
\hline Grupos & Media & Varianza & $\begin{array}{l}\text { Desviación } \\
\text { típica }\end{array}$ & $\begin{array}{l}\text { Valor } \\
\text { mínimo }\end{array}$ & $\begin{array}{l}\text { Valor } \\
\text { máximo }\end{array}$ & Curtosis & Asimetría \\
\hline $\begin{array}{l}\text { Adolecentes } \\
\quad(\mathrm{n}=53)\end{array}$ & 91,9 & 91,9 & 8,8 & 67,9 & 100 & 6,1 & $-0,9$ \\
\hline $\begin{array}{c}\text { Adultas } \\
\text { Primíparas } \\
(\mathrm{n}=53)\end{array}$ & 96,1 & 48,8 & 6,9 & 67,9 & 100 & 7,1 & $-2,6$ \\
\hline $\begin{array}{l}\text { Adultas } \\
\text { multíparas } \\
(\mathrm{n}=53)\end{array}$ & 94,2 & 57,5 & 7,6 & 64,4 & 100 & 5,7 & $-2,1$ \\
\hline
\end{tabular}




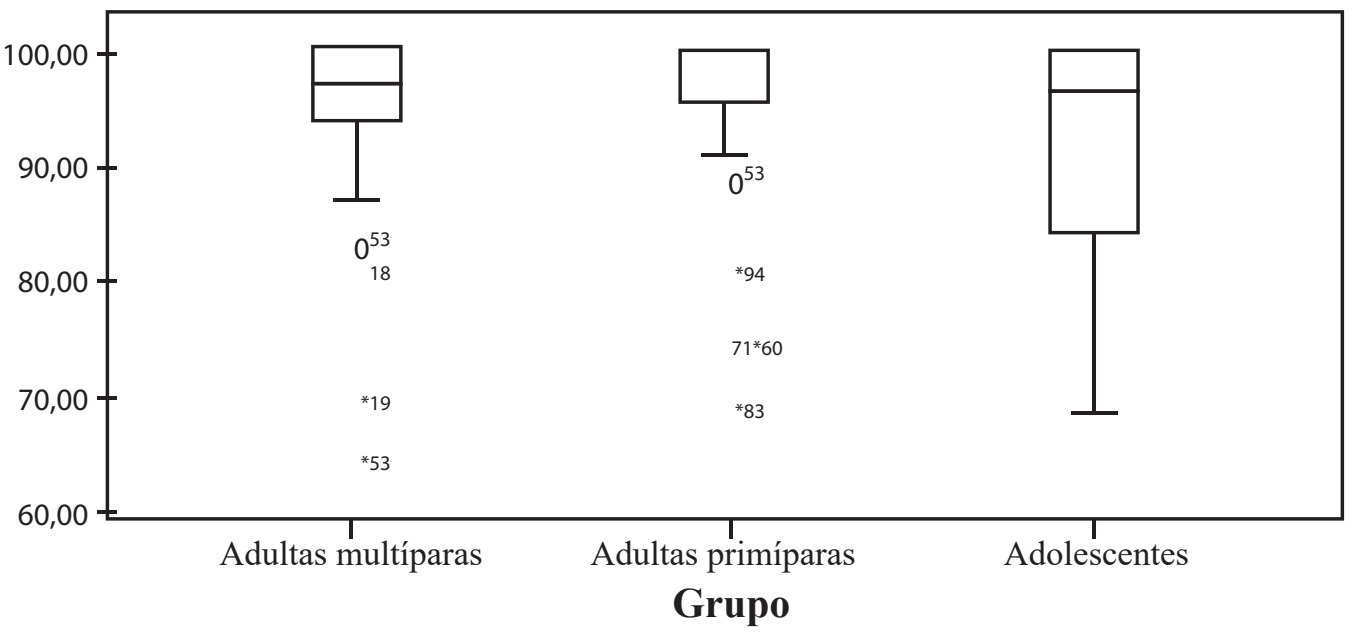

Figura 3. Comparación del vínculo afectivo en la dimensión Unión-interacción en los grupos adolescentes, adultas primíparas y adultas multíparas, Ecuador, Provincia de Bolívar.

está en el grupo de adultas primíparas, seguido de las adultas multíparas y de las adolescentes, con una mayor varianza en este último grupo. La curtosis demuestra que la concentración de los valores alrededor de la media a favor del grupo interacción de los tres grupos, al compararlos, revelan que la media más alta adultas primíparas, seguido de las adultas multíparas. Por el contrario, el grupo adolescentes que presenta una media más baja, con mayor dispersión de los datos.

El diagrama de la Figura 3 da cuenta de la distribución de los datos en la dimensión Unión-interacción, que muestra la posición de la media en los tres grupos, con un resultado aventajado para el grupo adultas primíparas.

\section{Discusión}

Desde la década de los ochenta las interacciones entre madres adolescentes y adultas con sus hijos han sido estudiadas, los resultados demuestran que las madres adultas revelaron más comportamientos con afectos positivos hacia sus hijos que las madres adolescentes (Bornstein et al., 2012). Del mismo modo, esos resultados podrían complementar los del presente estudio en cuanto a la valoración que expresaron las madres adultas y adolescentes del vínculo afectivo con sus hijos.

Estudios como los de Bornstein et al. (2012), Easterbrooks et al. (2005), Pomerleau et al. (2003) y el de Sánchez e Hidalgo (2002) presentan evidencias que concuerdan con los resultados de nuestro estudio. Por ejemplo, Pomerleau et al. (2003) indican que madres adolescentes en comparación con las madres adultas vocalizan menos con sus hijos, tienen una estabilidad moderada a través del tiempo resultados asociados con la actuación del infante a los seis meses en las escalas Bayley.

Por su parte, el estudio de Sánchez et al. (2004) realizado con madres adultas multíparas, encontró que las madres 
valoraban comportamientos de vinculación con el recién nacido; por ejemplo, el contacto con su bebé, que lo califican como "beneficioso". Los resultados revelan una relación significativa entre el grado de satisfacción materna $(P<0,001)$ y el tiempo de contacto piel a piel con su hijo al nacer. Una mujer adulta puede iniciar el deseo de tener a su hijo y una vinculación precoz, y que con poca frecuencia ocurre en la madre adolescente que no planifico su embarazo. Los resultados del estudio realizado por Easterbrooks et al. (2005) con díadas de madres jóvenes indican una variabilidad en la disponibilidad emocional con sus bebés. Por su parte, Bornstein et al. (2012), muestran que las madres adolescentes exhibieron un comportamiento materno menos favorable en lo verbal y sensibilidad. Al respecto, la adolescencia es una etapa de cambios a nivel físico, emocional, cognitivo y sensorial y empezar un embarazo genera la interrupción del desarrollo, obligándola a madurar presurosa por ende al contacto con el recién nacido se percibe variabilidad emocional. Los resultados del presente estudio en la dimensión Apoyo emocional con respecto a la desventaja percibida por las adolescentes son coherentes con el resultado del meta-análisis de Clemmens (2001) que halló un tamaño de efecto medio y una relación significativa entre el apoyo social de las madres adolescentes y la interacción con sus bebés, por lo que cabe resaltar que el apoyo emocional es un fenómeno esencial en el desarrollo vincular de la díada.
La dimensión Unión-interacción del Vínculo afectivo padres e hijos neonatos que en el presente estudio también reportó una diferencia significativa, abarca comportamientos que mostraron la satisfacción de la madre al compartir con su hijo, factor determinante en la relación vincular. En relación con lo anterior, Bornstein et al. (2012) hallaron en su estudio que conductas infantiles como la expresión facial, la vocalización y la exploración actúan como expresiones conductuales del estado de alerta, así como del funcionamiento cognitivo, comunicativo, emocional y social en la dinámica del vínculo en la díada. Para estudiar los comportamientos en la interacción de la díada, Sánchez e Hidalgo (2002) desarrollaron una investigación, sus resultados señalan que las interacciones de las madres adultas eran más cálidas, sensibles y afines con el lenguaje y la autonomía de sus bebés y demostraron mayor organización con los eventos cotidianos, como el de dar alimento.

\section{Conclusiones}

La comparación de los tres grupos demostró que las participantes adultas valoraron un mayor vínculo afectivo con sus neonatos comparado con las madres adolescentes $P$ valor 0,02 . Con respecto a la paridad, el estudio muestra que no existe diferencia en paridad en los resultados emitidos frente a la valoración del vínculo afectivo, de ello da cuenta los grupos de madres adultas primíparas y multíparas cuyos resultados en todos los análisis fueron relativamente cercanos. El análisis por dimensiones arrojó diferencia 
significativa en las dimensiones Apoyo emocional $P=0,04$ y Unión-interacción $P=0,02$, a favor de las díadas de madres adultas primíparas con desventaja para las díadas de madres adolescentes. El impacto de la presente investigación es de tipo social al obtener resultados con evidencias del vínculo afectivo entre madre y su neonato por grupos según edad y paridad motivando a realizar intervenciones individualizadas para mejorar cada etapa de la persona y que al futuro sea demostrado que influye positivamente.

Los resultados indican que madres adolescentes requieren de mayor atención y apoyo social familiar. El desarrollo físico y psicológico podría manifestarse con expectativas de logros independientes a una maternidad, lo que repercute en la unión-interacción y apoyo emocional con el recién nacido, dimensiones que son significativas en la vinculación afectiva madre e hijo. Diferente a los resultados de madres adultas que asumen este rol con responsabilidad y en la mayoría de los casos por decisión propia.

\section{Referencias}

Bornstein, M. H., Suwalsky, J. T., y Breakstone, D. A. (2012). Emotional relationships between mothers and infants: Knowns, unknowns, and unknown unknowns. Development and psychopathology, 24(1), 113-123.

Carrillo, G, J., y Quispe, C. N. (2013). Relación entre factores psicosociales y nivel del vínculo afectivo en madres primíparas $y$ sus recién nacidos a término. Tesis de pregrado. Hospital Belén de Trujillo 2012. Universidad Privada Antenor
Orrego, Trujillo, Perú.

Council for international of Medical Sciences. (2016). International ethical guidelines for health-related research involving humans. Geneva: Council for International Organizations of Medical Sciences. Recuperado de:

https://cioms.ch/wp-content/uploads/2017 /01/WEB-CIOMS-EthicalGuidelines.pdf

Clemmens, D. (2001). The relationship between social support and adolescent mothers' interactions with their infants: A meta-analysis. Journal of Obstetric, Gynecologic, \& Neonatal Nursing, 30(4), 410-420.

Easterbrooks, M. A., Chaudhuri, J. H., y Gestsdottir, S. (2005). Patterns of emotional availability among young mothers and their infants: A dydaic, contextual analysis. Infant Mental Health Journal: Official Publication of The World Association for Infant Mental Health, 26(4), 309-326.

Farkas, C., Santelices, M. P., Aracena, M., y Pinedo, J. (2008). Apego y ajuste socio emocional: Un Estudio en Embarazadas Primigestas. Psykhe, 17(1), 65-77.

Rodríguez, M. F. (2013). La autonomía emocional. Revista de Claseshistoria, (5), 2.

Garrido, M., y Marchán, M. (2011). Adopción del rol materno en madres adolescentes primerizas según grupo de conveniencia. Revista de Psicología, 13 (1), 11-28.

Meighan, M. (2011). Ramona T. Mercer: Adopcion del Rol materno - convertirse en madre. En M. Raile Alligood, A. Marriner Tomey, \& Mosby (Ed.), Modelos y teorías en Enfermería (Séptima ed., p. 581-598). Barcelona, España: Elsevier.

Ministerio de Salud Pública del Ecuador. (2010). Normas, protocolos y consejería para programas de atención nutricional durante 
el embarazo y parto. Recuperado de: http://docplayer.es/377661Normas-protocolos-y-consejeria-para-pro gramas-de-atencion-nutricional-durante-e l-embarazo-y-parto.html

Moreno Mojica, C., Rincón Villa Mil, T., Arenas Cárdenas, Y. M., Sierra Medina, D., Cano Quintero, Á. P., y Cárdenas Pinzón, D. L. (2014). La mujer en posparto: un fenómeno de interés e intervención para la disciplina de enfermería. Revista Cuidarte, 5(2), 739-747.

OPS, OMS. (2016). Pensamiento saludable: Manual para la atención psicosocial de la depresión perinatal. Recuperado de: http://iris.paho.org/xmlui/handle/123456 789/28417.

Pomerleau, A., Scuccimarri, C., y Malcuit, G. (2003). Mother-infant behavioral interactions in teenage and adult mothers during the first six months postpartum: Relations with infant development. Infant Mental Health Journal: Official Publication of The World Association for Infant Mental Health, 24(5), 495-509.

República de Colombia, Ministerio de Salud. 1993. Resolución 8430 de 1993. Por la cual se establecen las normas cientificas, técnicas y administrativas para la investigación en salud. Bogotá, Colombia. Recuperado de:

https://www.invima.gov.co/images/pdf/te cnovigilancia/buenas_practicas/normativ idad/Resolucion_8430_de_1993.pdf

Crugnola, C. R., Ierardi, E., Gazzotti, S., y Albizzati, A. (2014). Motherhood in adolescent mothers: Maternal attachment, mother-infant styles of interaction and emotion regulation at three months. Infant Behavior and Development, 37(1), 44-56.

Rosero, E. M., \& Sandoval, M. C. (2016). Factores que determinan el apego de la madre multipara hacia el recién nacido hospitalizado en la sala de patología neonatal 205 del hospital Gineco Obstétrico Isidro Ayora. Tesis de posgrado. Universidad Central del Ecuador, Quito Ecuador.

Sánchez, A., Martínez, M.T., y Hernández, M..C. (2004). El contacto precoz madre - hijo en el posparto: satisfacción materna y vínculo afectivo. Matronas profesión, 5(15), 12-18.

Sánchez, J., \& Hidalgo, M. (2005). Madres adultas $y$ madres adolescentes. Un análisis comparativo de las interacciones que mantienen con sus bebés. España, Sevilla. Recuperado de: http://copao.cop.es/files/contenidos/VOL 20_2_5.pdf

Universidad Técnica Particular de Loja (UTPL), Instituto Latinoamericano de la familia (ILFAM); Corporación para el desarrollo de la familia - (ORIENTAR). (2016). Familia en cifras 2016. Recuperado de: http://redifam.org/wp-content/uploads/20 17/05/ILFAM-ecuador-publicaci $\%$ C3\%B 3n01-texto.pdf.-Ecuador-familia-en-cifas -2016.pdf

Vargas, A. N. (2016). Validación del instrumento: "vínculo entre padres e hijos neonatos" a partir del Maternal Attachment Inventory. Tesis de posgrado. Facultad de Enfermería. Universidad Nacional de Colombia, Colombia. 
